\title{
Plant Disease and Defence
}

\author{
Steffen Rietz, Max Planck Institute for Plant Breeding Research, Cologne, Germany \\ Jane E Parker, Max Planck Institute for Plant Breeding Research, Cologne, Germany
}

Pathogens have evolved elaborate mechanisms to invade plant tissues and cause disease. Plants, in turn, respond to pathogen infection by expressing multiple layers of resistance. Once low energy 'basal' defences of plant cells have been overcome by pathogens, the plant can employ a more energy costly system of nonself recognition that triggers an 'acute' immune response. The survival of plants depends on their ability to fine-tune defence responses to the type of pathogen they encounter.

\section{Introductory article}

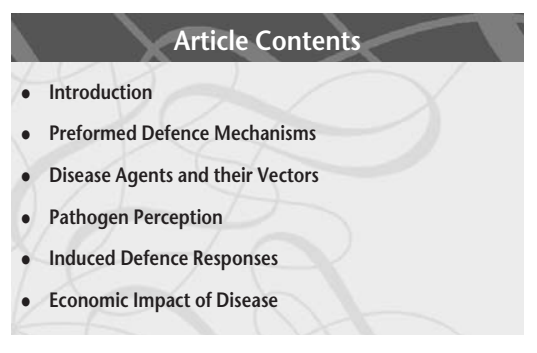

doi: 10.1002/9780470015902.a0004036

\section{Introduction}

From the germination of their seed, plants grow in one place and are therefore unable to escape unfavourable environmental conditions. Any circumstance that affects normal growth and development can be described as disease. The causes of disease are many and can be divided into biotic and abiotic stresses. The latter originates from soil (e.g. nutrient supply), climate (e.g. temperature) and pollution (e.g. ozone), while biotic stresses are inflicted by parasitic organisms that live off the plant. Through evolution viruses, microbes and animals have developed mechanisms to access plant resources. If this becomes detrimental to the plant, such organisms are known as pathogens. Plants, in turn, have evolved strategies to counter pathogen attack and these are expressed both as preformed barriers and induced defences. Such a multilayered defence system confers effective resistance on the majority of plants to most pathogens and their interaction is known as 'incompatible'. Once a pathogen has evolved the capability to overcome plant defences, the interaction is 'compatible'. Incompatibility (resistance) and compatibility (disease) are not absolutes but more of a continuum that arises from pathogen and plant diversification in an evolutionary 'arms race' of attack and counter-attack. Plant inducible defences can be expressed by individual cells and, as such, are comparable to the innate immune response of animals. Indeed, some of the underlying principles of animal and plant innate immunity are the same and in both systems the rate and amplitude of transcriptional activation of defence genes appear to be decisive in controlling the extent of local and systemic immune responses. In this review we describe the various causes of plant disease and our current understanding of plant resistance mechanisms. Our focus is on biotic disease agents, in particular microbial pathogens and nematodes. The effects of abiotic stress and of insect or animal feeding are only cursorily mentioned here and are covered well in other reviews. See also: Plant Defences Against Herbivore and Insect Attack; Plant Salt Stress; Plant Response to Water-deficit Stress

\section{Preformed Defence Mechanisms}

Preformed defences are present in healthy plants and constitute a major deterrent to pathogens. The nature of chemical and physical barriers to disease varies between different plant species and in some cases has evolved in response to particular pathogens in a plant's environment. As a consequence, preformed defence mechanisms account for resistance to the vast majority of parasitic microorganisms and play an important role in the expression of so called nonhost or species level resistance.

\section{Physical barriers}

To feed on nutrients in intercellular spaces and within cells of plants, pathogens must first breach the epidermis of leaves or the rhizodermis of roots and their associated physical barriers. The epidermis is a monolayer of cells interrupted by stomates that allow regulated gas exchange needed for transpiration and carbon fixation. The epidermal cell wall secretes cutin and waxes as an aliphatic film (cuticle), which protects the plant from desiccation and nutrient loss but also serves as a barrier against microbes and insects. The cuticle is a complex mixture of crosslinked lipids and its hydrophobic nature repels water and can impede fungal spores from adhering to and germinating on the leaf surface. Plant cells themselves are surrounded and shaped by a cell wall consisting of cellulose, hemicellulose and pectin. These compounds can be supplemented by lignin to support physical strength in water conducting tissue or layers of suberin to confer water impermeability in the casparian strip of the root. Beyond the cuticle, the physical rigidity and strength of the cell wall is not readily overcome and only pathogens that encounter a wound site or have evolved mechanisms to breach these layers are able to invade. See also: Plant Waxes; Plant Cuticle and Suberin; Plant Cuticle

Certain plants have epidermal cells that develop into specialized structures (such as trichomes), which impede insect movement or can secrete toxic chemicals. Other plants have spines or prickles (e.g. cacti) derived from 
epidermal or subepidermal cell layers to repel animals and insects. The thorns of roses serve the same purpose but originate from leaves by shoot metamorphosis. During secondary growth, the roots and stems of woody plants form an even more resistant tissue called the periderm. Meristematic cells (phellogen) differentiate several layers of cork cells, which die and form an outer bark. Penetration of the bark is rare and the majority of pathogens attacking the underlying tissues rely on gas channels (lenticels) or wound sites to gain entry.

\section{Constitutive chemical defence}

An impressive feature of plants is their repertoire of secondary metabolites (compounds that have no immediate function in primary metabolism such as photosynthesis, respiration or replication). Many secondary molecules, however, influence plant-pathogen interactions. The amounts of defensive secondary metabolites that accumulate constitutively in plant tissues vary and often low levels in healthy plants are boosted in response to pathogen challenge. This strategy saves energy for plant growth and reproduction in a competitive environment and has the advantage of creating a rapidly mobilizable defence system upon pathogen attack (see the section on Induced Defence Responses). Structurally diverse plant toxins can be broadly classified into three groups: terpenes, phenolic compounds and nitrogen-containing compounds. Terpenes, such as gossypol from cotton, are lipid molecules that deter a large number of plant-feeding insects and animals. Saponins belong to the same class and act against fungal pathogens by causing loss of membrane integrity by complexing sterols. Tannins are phenolic compounds that have been shown to reduce growth and survival of many herbivores by inactivating digestive enzymes and creating complexes with plant proteins that become difficult to digest. Alkaloids are a well-studied group of nitrogencontaining plant secondary metabolites due to their potent pharmacological properties in vertebrates. Common examples are morphine (from Papaver somniferum), cocaine (Erythroxylum coca) and nicotine (Nicotiana tabacum). Molecularly, their modes of action are variable, although many interfere with the nervous system or affect membrane transport. See also: Plant Alkaloids; Terpenoids: Higher; Terpenoids: Lower

Since many defence compounds are also toxic to the plant itself, they are restricted to certain organs such as glandular trichomes or are stored in cell compartments as inactive precursors. For example, glycosinolates of cruciferous plants become activated by plant myrosinase enzymes. In intact cells glycosinolates and myrosinases are spatially separated and upon tissue damage come together to release highly reactive molecules that are toxic to fungi and most herbivores. A good example of evolutionary specialization to a host is that of Pieris spp. (cabbage butterfly) that are adapted to feed on Cruciferae and whose feeding activity can even be stimulated by glucosinolates. The plant arsenal of preformed defences also contains proteins that inhibit pathogen hydrolytic enzymes, thereby impeding plant cell degradation during pathogen attack. A further battery of antimicrobial proteins is produced after a pathogen challenge, as discussed in the section on Induced Defence Responses. See also: Glycosides: Naturally Occurring

\section{Disease Agents and their Vectors}

Disease is an alteration of plant physiology that disturbs the performance of its vital functions and is usually diagnosed by specific symptoms. Common visual symptoms are wilting, yellowing (chlorosis) or dying (necrosis) of leaves and can extend to complete growth failure and death. Often the environmental circumstances affect plant vitality and can support pathogen ingress. The majority of biotic diseases are caused by viruses, bacteria, fungi and oomycetes (a branch of pathogens that are similar to fungi but more closely related to algae) that together can be referred to as microbes. Nematodes and chewing or probing insects also have significant impact on plant vitality. In plant-microbe interactions, the plant becomes a 'host' when the pathogen overcomes plant defences and propagates on the plant.

Microbial pathogens can be divided into biotrophs and necrotrophs according to their lifestyles. At one extreme, obligate biotrophic pathogens have evolved sophisticated mechanisms to parasitize living cells, minimize damage and perpetuate host cell viability until they can complete their life cycle. An effective resistance response of plants to these pathogens is local plant cell suicide (see the section on Pathogen perception). At the other extreme, necrotrophic pathogens often require wound sites or dying tissues to invade. They then rapidly macerate host cells and derive nutrients from dead tissues to spread. Many pathogens, however, fall between these two extremes and there are good examples of hemi-biotrophic pathogens, such as the potato late blight fungus Phytophtora infestans that invades as a biotroph before switching to necrotrophy - with devastating results. The biology of different pathogens illustrates well their diversity and ingenuity in causing disease.

\section{Viruses}

Viruses are simple entities of nucleic acid surrounded by a protein coat or by lipoprotein membrane. Their size is in the range of $10-250 \mathrm{~nm}$ and is therefore several orders of magnitude smaller than a plant mesophyll cell (Figure 1). Viruses are obligate parasites since they rely entirely on the replication machinery and metabolism of the host cell to propagate. Important features in virus classification are their shape and nucleic acid type. Viral genomes consist either of a single or double stranded deoxyribonucleic acid (DNA) or ribonucleic acid (RNA) that encode components necessary to replicate a complete virus particle. Viroids are molecularly even simpler than viruses in that they lack a protein envelope. They consist only of nonprotein coding 


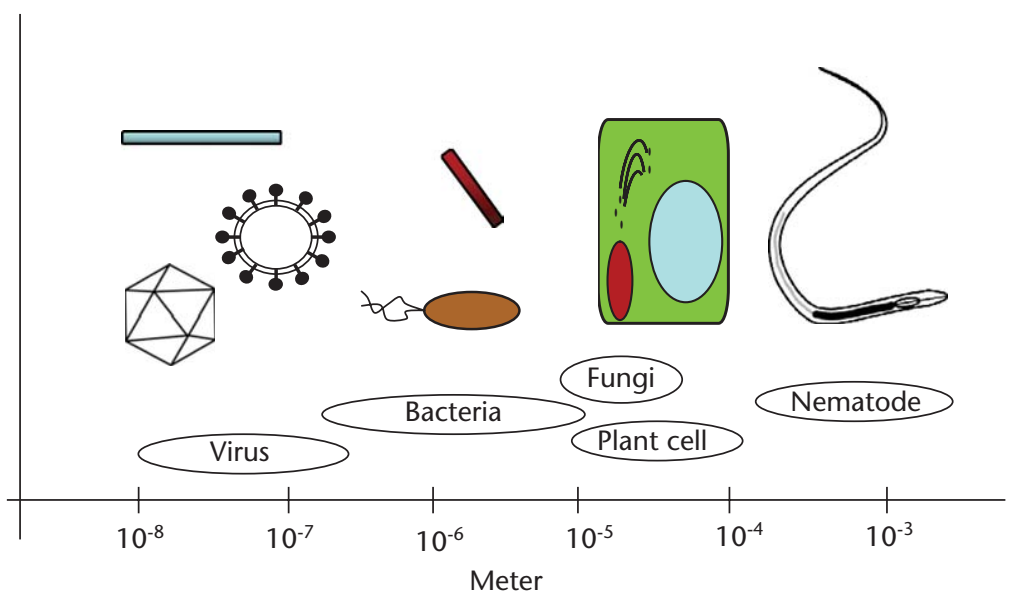

Figure 1 Sizes and shapes of different pathogenic microorganisms and nematodes in relation to the plant cell.

single-stranded circular RNA and can cause symptoms that are similar to a viral infection (see below) but are poorly understood.

Viruses infect plants through mechanical or vector transmission since they have no means by themselves to move or breach plant surfaces. Mechanical transmission can be achieved through wounds or by grafting. The principle method of spread is, however, by insect or nematode vectors that take up virus particles while feeding, often leading to severe economic losses. Once a virus reaches the cytosol it starts to replicate, exploiting the host's resources. To spread to neighbouring cells and eventually throughout the whole plant, viruses traverse plasmodesmata using viral-encoded movement proteins and the symplastic (cell-to-cell) transport machinery.

Certain viruses infect a plant without producing obvious signs of disease while others cause rapid death of the whole plant. Between these extremes there is a wide variety of symptoms in particular hosts, reflected by the names given to viruses. For example, Barley yellow dwarf virus (BYDV) forms chlorotic leaf symptoms on barley (Hordeum vulgare) and the Tobacco mosaic virus (TMV) forms mottled spots of necrotic cells on tobacco leaves (Nicotiana tabacum). BYDV is transmitted by aphids, while TMV spreads through mechanical transmission.

Viral infection invokes physiological changes in the plant cell that often lead to a decrease in the rate of photosynthesis, an increase in respiration and changes in the activities of plant growth regulators. Plants have in turn evolved multiple defence strategies. Certain plant genotypes have evolved immune receptors (also known as Resistance proteins) that specifically recognize a viral component and trigger cell death (a hypersensitive response, HR) at the site of inoculation (see the section on Pathogen perception). This effectively prevents viral propagation and is accompanied by the local and systemic up-regulation of genes encoding defence proteins (also known as Pathogenesis-Related or PR-genes, see the section on Induced Defence Responses). Plants also employ an elaborate system of RNA silencing to target the viral genome. RNA silencing or RNA interference (RNAi) occurs naturally in plant and animal cells to regulate the abundance of RNAs and has been recruited to recognize and destroy foreign RNA molecules. In a beautiful twist of evolutionary counter-defence, some viruses have learned to suppress the RNAi machinery and thereby reassert their infectivity.

\section{Bacteria}

Bacteria are unicellular prokaryotic organisms that exploit almost every environmental niche on earth and have evolved sophisticated tools to overcome plant defence and cause disease. Bacterial symptoms can be divided into four categories:

1. Yellowing or chlorosis by toxins (e.g. Pseudomonas spp.).

2. Wilting due to clogging of vascular (transportation) tissue (e.g. Xanthomonas spp.).

3. Maceration of tissue through lytic enzymes (e.g. Erwinia spp.).

4. Abnormal cell division by distorted plant hormone balance (e.g. gall formation by Agrobacterium tumefaciens).

Pathogenic bacteria are not able to cross intact plant surfaces and entry is normally via natural openings (such as stomates) or wounds (Figure 2). The bacteria multiply in intercellular spaces or xylem vessels, exploiting the massive nutrient resources of the host. Many phytopathogenic bacteria (e.g. Pseudomonas, Erwinia) inject effector proteins (also known as virulence factors) into host cells by a type III secretion system (TTSS) to cause disease. Once the bacterium contacts the plant cell it forms a channel-like structure (a pilus) that penetrates the host cell wall and releases effector proteins into the cytosol. An expanding body of genomic information on bacterial pathogens 


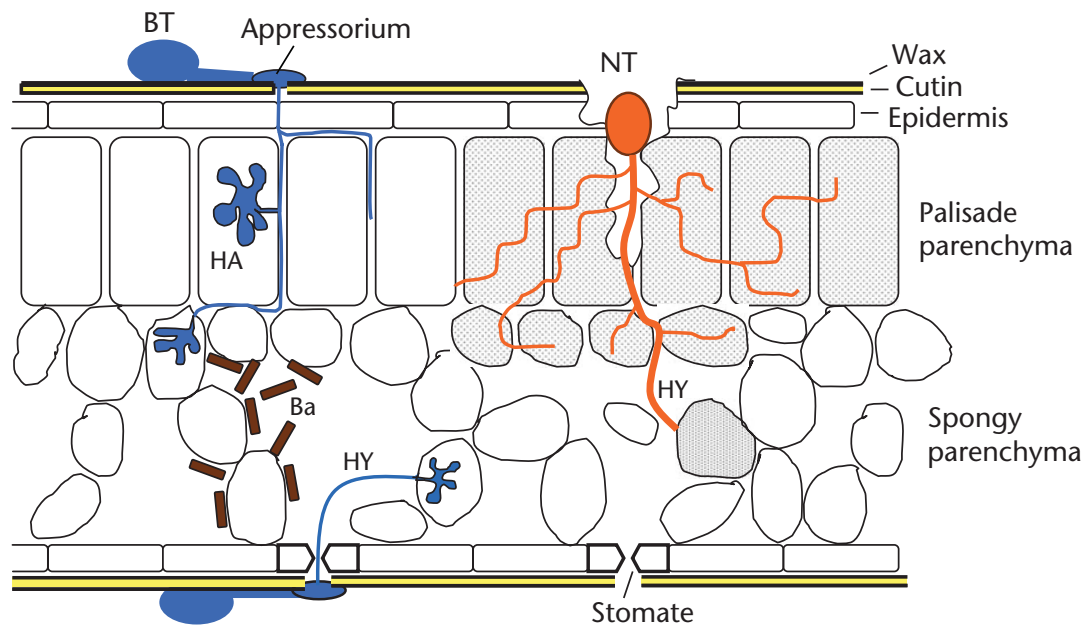

Figure 2 Leaf cross-section showing intercellular bacteria (Ba) and biotrophic (BT) or necrotrophic (NT) fungi/oomycetes. HA: Haustorium, HY: Hyphae.

reveals that secreted effector proteins help to interfere with various layers of plant cellular defence by, for example, modification or proteolytic cleavage of host proteins. Again, some plant genotypes are resistant to particular bacterial strains due to the expression of immune receptors that recognize specific effectors. However, the rapid evolution of bacterial genes to avoid recognition means that this mode of resistance is normally not durable.

\section{Fungi and oomycetes}

Fungi and oomycetes are eukaryotic organisms that span the range of biotrophy to necrotrophy. Infection is initiated when a spore lands on the plant surface and senses environmental conditions (e.g. humidity, temperature) and the presence of a suitable host plant before germination. Under appropriate conditions a germ tube grows along the plant surface and forms an infection structure (appressorium or infection peg) that allows the pathogen to penetrate epidermal cells (Figure 2). Breakage of the plant cell wall is an active process generating enormous turgor pressure at the appressorial-cell interface and the secretion of lytic enzymes such as cutinases, pectinases and cellulases to degrade the cell wall. Biotrophs form feeding structures (haustoria) within host cells that invaginate the plasma membrane and therefore extend the surface area through which nutrients can be absorbed for growth and signals exchanged between host and pathogen. Mycelia then extend intercellularly or on the plant surface, causing minimal disruption to the host plant before making of the next generation of spores. See also: Plant Defences Against Fungal Attack: Biochemistry

Rapid host cell death during an HR effectively ends infection by a biotrophic pathogen. In contrast, necrotrophs such as the grey mould fungus (Botrytis cinerea) thrive on dead cells. Its invading hyphal peg does not form a haustorium but releases phytotoxic metabolites that induce a cell death programme. Growth of Botrytis was found to be suppressed on a host impaired in initiation of an HR but stimulated by simultaneous inoculation with an HRinducing bacterium. Thus, while plant cell suicide is an efficient mechanism to fight biotrophic pathogens it must be very carefully regulated to avoid succumbing to necrotrophs.

\section{Nematodes}

Nematodes (or roundworms) are multicellular animals that are circular in cross-section and elongated at each end with an average size of $0.5-10 \mathrm{~mm}$ (Figure 1). Many are free living in soil and water, reproduce sexually and feed on detritus. In suitable environmental conditions, the nematode hatches from an egg and develops through four juvenile stages to become an adult. Plant parasitic nematodes are obligate biotrophs that feed only on living cells. The two most damaging groups that affect crop species worldwide are the soil-dwelling root knot nematodes (Meloidogyne spp.) and cyst nematodes (Heterodera and Globodera spp.). They are both sedentary endoparasites of plant roots and have similar life cycles. The first juvenile stage hatches in the parental body and the second and infectious stage (J2) hatches in the soil. The $\mathrm{J} 2$ nematodes penetrate host roots and move to the plant vascular system to start feeding. To puncture the plant cell wall, parasitic nematodes possess a hollow axial spear (stylet) in the oral aperture that also secretes into the cell and absorbs its cytoplasmic contents. Root knot nematodes induce the formation of enlarged and multinucleated giant cells at feeding sites while cyst nematodes initiate expansion of the initial feeding cell giving rise to a cyst (syncytium). Macroscopically, the formation of galls or cysts along the root are characteristic of nematode infection and often stunting and yellowing of shoots that ensues can be especially devastating to yields in crops such as corn, potato, soybean and sugar beet (http://nematode.unl.edu/). Plants protect themselves from nematodes through chemical and physical barriers as well as specific resistance mediated by immune receptors (see the section on Pathogen perception). 


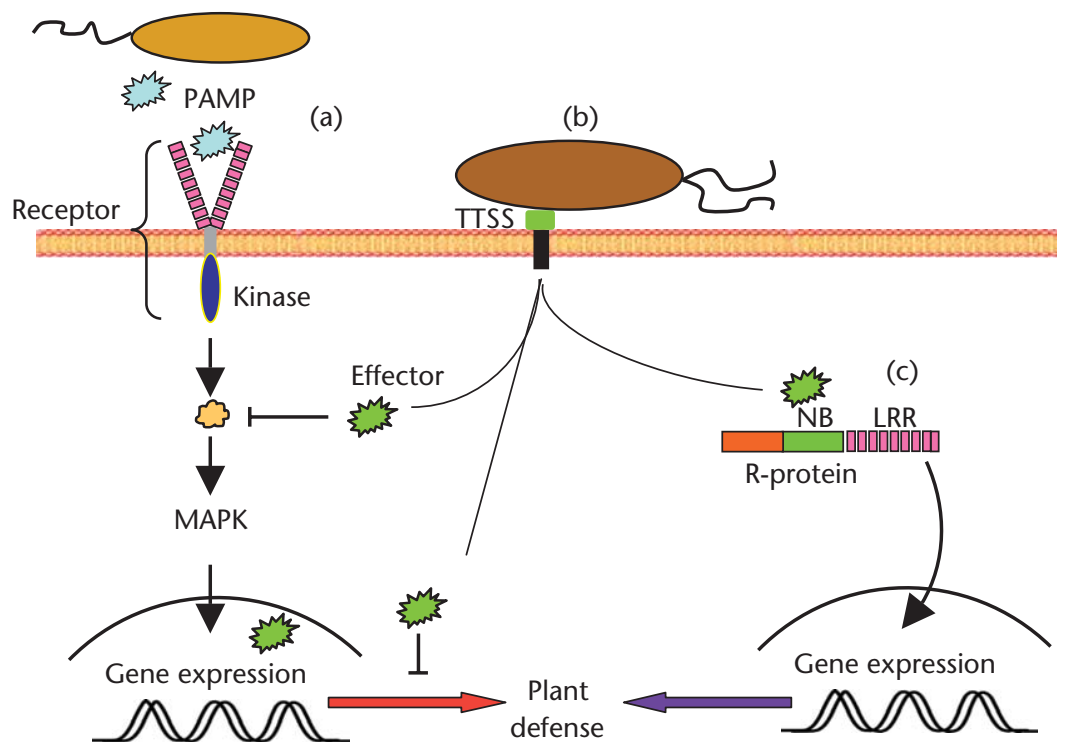

Figure 3 Evolution of the plant-bacterial pathogen interaction. (a) The plant has evolved receptors to sense pathogen associated molecular patterns (PAMPs) that trigger basal defences. (b)The bacterium injects effector proteins into the plant cell via a type III secretion system (TTSS) to interfere with defence signalling or response. (c)The plant responds to infection by the generation of immune receptors encoding nucleotide-binding (NB), mitogen-associated protein kinase (MAPK), leucine-rich-repeat (LRR) R-proteins that recognize effectors and trigger an acute defence response usually involving cell death.

\section{Pathogen Perception}

Sensing of pathogens is crucial for the survival of the plant. During ingress, a pathogen cannot avoid interacting physically with the plant and it also needs to release chemicals that facilitate cuticle and cell-wall digestion. Plants have evolved to recognize these nonself molecules by the expression of a battery of receptors, many of which lie in its plasma membrane and are part of a general surveillance system. Pathogen-derived molecules as diverse as (poly)peptides, oligosaccharides or lipids that are sensed by the plant and trigger defence are called elicitors. The plant itself can produce endogenous elicitors that fine tune its interaction with pathogens. Microbial elicitors that trigger innate immune responses in plants and animals are termed 'Pathogen-Associated Molecular Patterns' (PAMPs) and the proteins that perceive them, PAMP receptors. Particular PAMPs are conserved within a pathogen species or genus and are not easily dispensed with so that PAMP recognition is a quite durable form of resistance. The extent of PAMP recognition in plants is not known but at least two plasma membrane-bound receptors that have leucine-richrepeat (LRR) interaction domains sticking out of the cell have been defined in the model plant Arabidopsis. They recognize, respectively, part of the bacterial flagellin protein and an elongation factor TU. These receptors have a phosphorylation (kinase) domain inside the cell that transduces the recognition signal and initiates downstream processes constituting a low level 'early warning' (or basal) immune response (see the section on Economic impact of disease).

Evidence is emerging from the study of plant-bacterial interactions that bacterial effectors exported into plant cells (See the section on Disease agents and their vectors) can aid infection by interfering with PAMP receptor-mediated 'basal' immune responses (Figure 3). Thus, effectors can act as virulence factors that suppress host defences at the cell surface, allowing the pathogen to gain entry and cause disease. Such an aggressive strategy by the pathogen drives the selection of plant immune receptors (introduced in the section on Disease agents and their vectors) that detect specific effector molecules. This form of nonself recognition can occur outside the cell, although the majority of immune receptor (R) proteins identified so far are expressed intracellularly. These are large modular proteins that have a LRR domain connected to a central nucleotide-binding/ oligomerization and a signal transduction domain and resemble, to some extent, pathogen-sensing receptors recently discovered in mammalian cells. Whether recognition of the pathogen effector is direct as a ligand binding its receptor or indirect by detecting the action of an effector on a particular host target, depends on the $\mathrm{R}$ protein-effector combination. Examples of both types of interaction exist. In the latter scenario, an $\mathrm{R}$ protein could 'guard' several pathogen targets thereby broadening the recognition capabilities of a limited number of host plant receptors. Immune-receptor activation by an effector triggers an 'acute' resistance response that normally culminates in cell death (the HR) at infection sites.

\section{Induced Defence Responses}

Inducible processes are crucial to express effective resistance at the right moment and to conserve energy for plant survival. In basal immunity, cell-wall reinforcements and mobilization of antimicrobial metabolites are needed to 
check pathogens at the cell surface. In effector-triggered immunity, initiation of the HR and its associated cell death programme are necessary to stop further invasion by hostadapted biotrophic pathogens. Local defence also triggers the mobilization of secretory pathways and secondary metabolites needed to induce systemic resistance. All of these processes require genetic reprogramming of the plant cell and in the model plant Arabidopsis thaliana, the expression of approximately $20 \%$ of its genes is changed upon pathogen attack. What appears to be important is the timing of these inducible defences. Thus, a virulent pathogen strain that can cause disease triggers qualitatively similar transcriptional changes to an avirulent pathogen, but with slower kinetics and lower amplitude. As important as induction is the delimitation of defence and cell death reactions and multiple negative regulators have been identified that allows the plant to save energy and exert fine-control of metabolism.

\section{Signal transduction}

Defence signal relay in the cell is organized into cascades of proteins that channel pathogen perception to a defence output. Generally, transduction pathways allow the plant to translocate a signal over a distance (e.g. from the cytoplasm to the nucleus or from one cell to another) and to amplify or reinforce the primary (elicitor) signal (Figure 4). A complex system of inter-connected pathways has evolved that permits exquisite tuning of the plant response depending on the type of pathogen perceived and the prevailing environmental conditions.

The earliest signalling events such as membrane depolarization, an increase in cytosolic $\mathrm{Ca}^{2+}$ and the production of reactive oxygen species (ROS) and nitric oxide (NO) occur within seconds or minutes of perception of a pathogen elicitor. These molecules act as secondary messengers that modulate protein functions. A change in cellular reduction/oxidation (red/ox) status is caused by ROS generation inside and outside the cell and this not only serves as an important stress signal but can be directly toxic to an

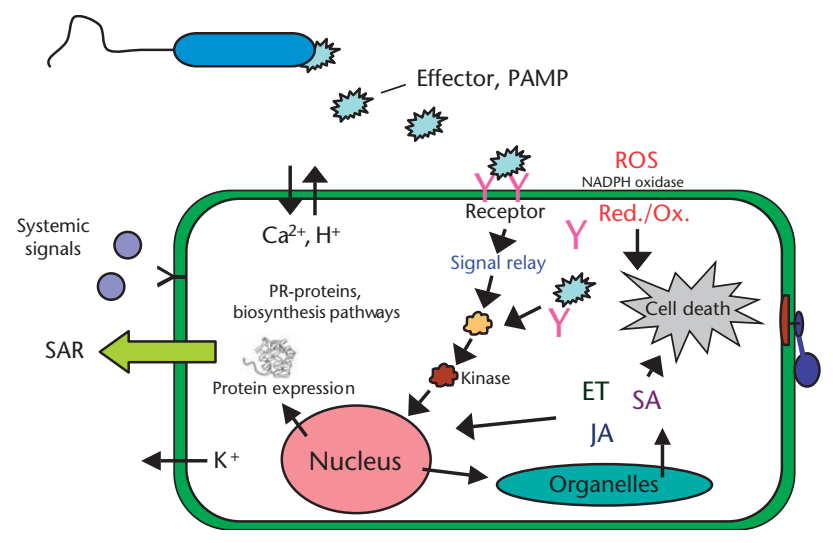

Figure 4 Plant signal transduction pathways in response to pathogen attack. ROS: reactive oxygen species, ET: ethylene, JA: jasmonic acid, SA: salicylic acid, SAR: systemic acquired resistance. invading pathogen by the oxidation of organic molecules. Predominant components of downstream signalling are protein kinases such as mitogen-associated protein kinases (MAPKs) and calcium-dependent protein kinases (CDPK) that transfer phosphate groups from adenosine triphosphate (ATP) to specific substrates. Often kinases act in sequence and become activated or repressed themselves upon phosphorylation as part of the signal relay (Figure 3). Although the final targets of kinase cascades remain elusive, they culminate in the transcriptional regulation of defence genes, in some cases, by active mobilization of transcription factors from the cytosol to the nucleus.

Transcriptional reprogramming of phytohormone biosynthetic and response pathways leads to a change in hormonal status throughout the plant and this is important to direct the local and systemic defences. Increased levels of the phenolic hormone salicylic acid (SA) signals in local resistance to invasive biotrophs and is necessary for systemic signal relay against these pathogens. In contrast, elevation of the phytohormones jasmonic acid (JA) and ethylene (ET) activates defences against insect wounding or attack by necrotrophic pathogens. Sites of crosstalk between these opposing defence systems have been identified that allow the plant to tailor its defences appropriately. See also: Systemic Signalling

\section{Induced antimicrobial factors}

The de novo synthesis of toxic secondary metabolites (known as phytoalexins) probably has a more important contribution in resistance to biotrophic and necrotrophic pathogens than has previously been appreciated. Structurally, many phytoalexins are isoflavonoids or sesquiterpenes and particular phytoalexin structures can be found in different plant families. The production of phytoalexins can be induced by elicitors and the growth and propagation of the pathogen inhibited in a dose-dependant manner. Thus, the introduction of phytoalexin biosynthesis genes in transgenic plants has in some cases increased their resistance to particular pathogens. See also: Secondary Metabolites: Killing Pathogens

The accumulation of a heterogeneous group of PR proteins in infected plants is also well documented. They are classified into 12 families based on relatedness between their amino acid sequences. For some, an antimicrobial or antiherbivore activity was demonstrated, while for others the biological function is not known. Other antipathogenic proteins were identified more recently that do not fit obviously into the PR-1 to PR-12 protein families. The patterns of these induced protein factors altogether referred to as induced antipathogenic proteins (iAPPs) vary depending on the types of microbes or abiotic stress and some are diagnostic for activation of the SA, ROS or JA/ ET pathways. Their expression is strongest at primary infection sites but up-regulation in uninfected tissues suggests involvement also in systemic resistance. Subcellularly, these proteins are mainly directed to the vacuole and are released after cell rupture or secreted to the apoplast where 
they may directly target pathogens. Some of the characterized iAPPs possess hydrolytic (e.g. chitinase or $\beta-1$, 3 -glucanase) enzyme activities that can digest pathogen structures. Other iAPPs with, as yet, poorly defined biochemical functions (e.g. thionins and lipid transfer proteins) act at the plasma membrane and are thought to affect lipid composition and membrane permeability. Clearly, more work needs to be done to clarify the roles of this diverse set of induced molecules in local and systemic immune responses and their precise modes of action.

\section{Economic Impact of Disease}

Crop production is the primary source of human and animal nutrition and other materials. Today, approximately $12 \%$ of the world population ( 820 million) is undernourished and increasing by a rate of four million a year (FAO). Factors that impact on the quantity and quality of crop yields, therefore, need to be carefully monitored and understood, and of these disease by microbial and insect pathogens are a major contributor to food loss. Assessing the extent of crop damage due to pathogens and pests is not trivial and in both developing countries and developed agricultural systems little effort is made to predict infestation or disease probabilities. In part, this arises from the difficulty in quantifying disease and relating this to achievable crop yields. Thus, blanket applications of chemicals are the norm and the increased cultivation of crop monocultures with a narrow genetic base increases the demand for pesticides and fungicides. There are a number of ways forward. Pathogens rapidly overcome single $R$ gene- (immune receptor) mediated host resistance that has been used successfully for short periods against prevalent pathogen races of several crops. Pyramiding of different $R$ genes in one variety may provide a more robust system since one pathogen variant is unlikely to build up to dangerous levels. This requires extensive breeding and molecular plant breeding tools have been impressively refined in recent years to permit rapid selection of desirable traits. This may also allow the introduction into high yielding crop varieties of low level but robust resistance traits such as those operating in 'basal' resistance described in this review. For this, however, we need to understand better how local and systemic defence circuits work so that enhanced resistance to one pathogen does not compromise the plant response to other pathogens or environmental stresses. Such strategies, combined with transgenic technology, the preservation of wild germplasms as a valuable resource of new genes and a better appreciation of pathogen population dynamics, may provide some direction for future disease control.

\section{Further Reading}

Alfano JR and Collmer A (2004) Type III secretion system effector proteins: double agents in bacterial disease and plant defense. Annual Review of Phytopathology 42: 385-414.

Chisholm ST, Coaker G, Day B and Staskauricz BJ (2006) Host-microbe interactions: shaping the evolution of the plant immune response. Cell 124(4): 803-814.

Delledonne M, Polverari A and Irene Murgia (2003) The functions of nitric oxide-mediated signaling and changes in gene expression during the hypersensitive response. Antioxidants \& Redox Signaling 5(1): 33-41.

Garcia-Brugger A, Lamotte O, Vandelle E et al. (2006) Early signaling events induced by elicitors of plant defenses. Molecular Plant-Microbe Interactions 19(7): 711-724.

Glazebrook J (2005) Contrasting mechanisms of defense against biotrophic and necrotrophic pathogens. Annual Review of Phytopathology 43: 205-227.

Grant M and Lamb C (2006) Systemic immunity. Current Opinion in Plant Biology 9(4): 414-420.

Nimchuk Z, Eulgem T, Holt BF and Dangl JL (2003) Recognition and response in the plant immune system. Annual Review of Genetics 37: 579-609.

Slusarenko AJ, Fraser RSS and Loon LC (2000) Mechanisms of Resistance to Plant Diseases. Dordrecht, The Netherlands: Kluwer Academic Publishers.

Zipfel C and Felix G (2005) Plants and animals: a different taste for microbes? Current Opinion in Plant Biology 8(4): 353-360. 